ISSN: 2386-3919 - e-ISSN: 2386-3927

DOI: https://doi.org/10.14201/et2018362145166

\title{
PROPUESTAS PARA ELEVAR LOS DEFICIENTES NIVELES ACADÉMICOS MASCULINOS
}

\author{
Increasing male low educational attainment
}

\author{
Mercedes VAROna Alabern \\ Florida Universitaria \\ Correo-e: varonamercedes@gmail.com
}

Recibido: 28/05/2018; Aceptado: 14/11/2018; Publicado: 30/12/2018

Ref. Bibl. MERCEDES VARONA ALABERN. Propuestas para elevar los deficientes niveles académicos masculinos. Enseñanza \& Teaching, 36, 2-2018, 145-166.

RESUMEN: La formación académica de la población condiciona la situación social, política y económica de un país. Por este motivo, los bajos rendimientos académicos de los varones hacen sonar todas las alarmas. Diversos estudiosos han abordado esta problemática. No obstante, su persistencia hacen necesaria la continua investigación para lograr solucionarla. El presente trabajo aborda este tema, planteando posibles soluciones. La metodología elegida para este cometido es descriptivo-explicativa, dado que permite resumir la precaria situación académica de los varones y, al mismo tiempo, profundizar en las causas de tales desigualdades, finalizando con propuestas tangibles para revertir esta tendencia. Se han encontrado cuatro principales causas responsables de entorpecer el éxito académico masculino. Las dos primeras están relacionadas con la falta de sensibilidad que los sistemas educativos manifiestan con las singularidades biológicas de ambos sexos. Estas disparidades afectan directamente el aprendizaje en el aula, dificultando el seguimiento de la instrucción por la totalidad del alumnado al no adaptarse a las necesidades individuales. Por otra parte, así como los animales poseen la mayoría de conocimientos para sobrevivir en sus genes, los humanos deben aprender casi todo a través de la imitación, de la reflexión y del estudio. Las dos últimas causas de los bajos rendimientos masculinos son la ausencia de la figura del padre en familias monoparentales o desestructuradas, con gravísimas consecuencias para los hijos varones, y la carencia de referentes masculinos en la escuela, lo que dejan a demasiados alumnos varones 
en un estado de gran vulnerabilidad, al no poder aprender imitando o al tener que buscar tales referentes en lugares poco idóneos. Por este motivo, el sistema educativo actual expulsa a un elevado número de individuos, haciendo necesaria una reevaluación del mismo que solucione las problemáticas aquí descritas.

Palabras clave: deficiencias académicas de los varones; niveles académicos masculinos; ausencia del padre; diferencias biológicas entre mujer y hombre; coeducación; educación diferenciada.

SUMMARY: Educational attainment shapes the social, political and economic outcome of a country. Therefore, male underachievement in education raises serious concerns, and several scholars have undertaken the task of reverting this tendency, but the problems still persist. Thus, further research is required. This paper delves into this predicament and proposes solutions. The method used is descriptive-explanatory because allows us to compile data about male low educational achievements. In addition, it allows us to examine the causes of male underperformance and give tangible and realistic proposals in order to revert this tendency. There are four main causes that impair male success in education. The first two are related to schools' lack of sensitivity regarding the relevant biological differences between girls and boys. These differences affect their learning process in the present educational system, making it harder for boys to succeed since the teaching methods are not adapted to their specific needs. On the other hand, unlike animals that have what they need for survival in their genes, humans learn everything by imitating their parents, their professors and their peers, and by studying and cogitating. The last two causes of male low academic performance are created by the absence of male role models at home, with dramatic consequences for boys, and at schools, as the majority of the teachers are female. This leaves male students very vulnerable because they are unable to learn by imitating valid male role models in school and at home, and they end up searching for them elsewhere. Consequently, a reform of the present educational system is proposed herein.

Key words: boys' underachievement in education; male academic performance; coeducation; single-sex education; father absence; biological differences between girls and boys.

\section{INTRODUCCIÓN}

Desde finales del siglo XIX la educación se ha ido perfilando como requisito imprescindible para que la sociedad avance y logre mantener estabilidad política, económica y social, convirtiéndose paulatinamente en un derecho. Sin embargo, el sistema educativo universal no ha logrado los éxitos que de él se esperaban, dado que un porcentaje elevado de escolares, compuesto en su mayoría por varones, no adquiere los conocimientos necesarios para poder salir adelante, poniendo en serio peligro no solo su futuro individual, sino también el colectivo. Son varias las investigaciones que han profundizando en este tema, constatando la influencia de 
las singularidades biológicas en el desarrollo académico y personal de la infancia (Cahill, 2005; Sax, 2005; Gurian, 2011; Dee, 2005; o Norfleet James, 2015), aunque hayan sido subestimadas por los defensores férreos de la coeducación, desaconsejando incluso su estudio (Eliot, 2011; Jordan-Young y Rumiati, 2011). Por este motivo, la grave situación académica de los varones persiste, evidenciando de este modo la incapacidad de resolver dicha problemática y la necesidad de continuar investigando para lograr elevar los niveles académicos masculinos, mejorando asimismo toda la sociedad. El presente trabajo ahonda en dicha problemática, analizando las deficiencias académicas de los varones en relación con las mujeres, detallando las causas de tales divergencias, aportando simultáneamente nuevas investigaciones, y finalizando con propuestas sólidas y plausibles para reducir el fracaso académico masculino.

\section{SitUACIÓN ACTUAL DE LA FORMACIÓN DE LOS VARONES}

Un aspecto que llama la atención por ser común a la mayoría de sistemas educativos de nuestro entorno es la supremacía académica de las mujeres en casi todos los indicadores. El abandono escolar vaticina el presente y futuro de un país y en España es alarmantemente elevado, siendo los varones quienes lo sufren con mayor crudeza (el 27,3\% no completó la Educación Secundaria Obligatoria en 2015, frente al 17,2\% de las mujeres) (Ministerio de Educación, Cultura y deporte, 2017: p. 89).

Más significativo es el retroceso de los jóvenes españoles frente a las féminas en las etapas de BUP y COU, invirtiéndose estos índices entre ambos sexos a lo largo del tiempo, favoreciendo a las mujeres (Tabla 1). Del mismo modo, el porcentaje de varones que obtiene la titulación de bachillerato o formación profesional descendió del 53,5\% en 1992, al 34 \%, en 2007 (Tabla 2), siendo las tasas para ambos sexos inequívocamente insostenibles para hacer frente a los retos políticos, económicos y sociales del siglo XXI (Tabla 3 ).

TABLA 1

Porcentaje de la población escolarizada en BuP y cOU por sexos. 1970-87

\begin{tabular}{|c|c|c|}
\hline \multirow{2}{*}{ AÑO } & \multicolumn{2}{|c|}{ BUP-COU } \\
\cline { 2 - 3 } & VARONES & MUJERES \\
\hline $1970-71$ & $54 \%$ & $46 \%$ \\
\hline $1975-76$ & $51 \%$ & $49 \%$ \\
\hline $1980-81$ & $47 \%$ & $53 \%$ \\
\hline $1985-86$ & $47 \%$ & $53 \%$ \\
\hline $1987-88$ & $47 \%$ & $53 \%$ \\
\hline
\end{tabular}

Fuente: Ministerio de EDUCACIÓN Y CienCIA. CIDE (1992: 18). 
TABLA 2

Porcentaje del abandono escolar temprano (sin finalizar bachillerato o equivalente) por sexo y grupo de edad. 1992 y 2007

\begin{tabular}{|l|c|c|c|c|}
\hline \multirow{2}{*}{ EDAD } & \multicolumn{2}{|c|}{ MUJERES } & \multicolumn{2}{c|}{ HOMBRES } \\
\cline { 2 - 5 } & 1992 & 2007 & 1992 & 2007 \\
\hline 18-19 años & $51,10 \%$ & $35,70 \%$ & $48,90 \%$ & $64,30 \%$ \\
\hline 20-21 años & $55,80 \%$ & $37,60 \%$ & $44,20 \%$ & $62,40 \%$ \\
\hline 22-24 años & $54,70 \%$ & $34,90 \%$ & $45,30 \%$ & $65,10 \%$ \\
\hline Total & $53,50 \%$ & $36,10 \%$ & $46,50 \%$ & $63,90 \%$ \\
\hline
\end{tabular}

Fuente: CASQuero y NAVARro (2010: 20).

TABLA 3

Porcentaje de la población entre 25-64 años con bachillerato o titulación equivalente, por países de OCDE. 2000, 2005, 2010, 2014, 2015 y 2016

\begin{tabular}{|l|c|c|c|c|c|c|c|c|c|c|}
\hline \multirow{2}{*}{ PAísES } & 2000 & 2005 & 2010 & 2014 & 2015 & \multicolumn{5}{c|}{2016} \\
\cline { 2 - 12 } & $25-64$ & $25-64$ & $25-64$ & $25-64$ & $25-64$ & $25-64$ & $25-34$ & $35-44$ & $45-54$ & $55-64$ \\
\hline OCDE & 65,1 & 70,6 & 74,4 & 77,1 & 77,2 & 78,4 & 84,1 & 81,8 & 77 & 69,7 \\
\hline Australia & 58,8 & 65 & 73,2 & 77,1 & 79 & 79,9 & 88,6 & 85,2 & 75,7 & 67,6 \\
\hline Austria & - & 76,9 & 82,4 & 83,9 & 84,6 & 84,5 & 88,6 & 87,2 & 83,5 & 78,6 \\
\hline Belgium & 58,5 & 66,1 & 70,5 & 73,6 & 74,7 & 75,1 & 82,8 & 81,5 & 75 & 61 \\
\hline Canada & 80,7 & 85,2 & 88,3 & 90 & 90,4 & 90,6 & 93,1 & 93,3 & 90,7 & 85,5 \\
\hline Czech Rep. & 85,9 & 89,9 & 91,9 & 93,2 & 93,2 & 93,4 & 93,4 & 95,4 & 94,6 & 89,6 \\
\hline Denmark & 79,8 & 81 & 75,6 & 79,6 & 80,4 & 80,7 & 83,4 & 85,3 & 81,1 & 73,2 \\
\hline Estonia & 85,2 & 88,7 & 89,1 & 88,1 & 88,6 & 88,9 & 87,5 & 86,6 & 93,6 & 88,2 \\
\hline Finland & 73,2 & 78,8 & 83 & 86,5 & 87,2 & 87,9 & 90,1 & 91,3 & 89 & 81,7 \\
\hline France & 62,2 & 66,8 & 70,8 & 77,3 & 77,5 & 78,1 & 86,7 & 84,2 & 76,7 & 65,2 \\
\hline Germany & 81,7 & 83,1 & 85,8 & 86,9 & 86,8 & 86,5 & 87 & 85,8 & 87,2 & 85,8 \\
\hline Greece & 49,3 & 57,7 & 62,7 & 68,3 & 70,2 & 71,7 & 84,5 & 78,2 & 69,4 & 54 \\
\hline Hungary & 69,2 & 76,4 & 81,3 & 83,1 & 83,2 & 83,4 & 85,5 & 86,5 & 83 & 78,1 \\
\hline Iceland & - & 68,2 & 70,7 & 73,3 & 74,7 & 78 & 80 & 81,3 & 79,3 & 70,7 \\
\hline Ireland & 57,3 & 64,5 & 72,8 & 78,8 & 79,8 & - & - & - & - & - \\
\hline Israel & - & 78,9 & 82,1 & 85,4 & 85,5 & 86,8 & 91,8 & 88,2 & 84,5 & 79,9 \\
\hline Italy & 42,1 & 50,1 & 55,2 & 59,3 & 59,9 & 60,1 & 73,9 & 65,7 & 54,9 & 48,4 \\
\hline Korea & 68,3 & 75,5 & 80,4 & 85 & 85,8 & 86,9 & 98,3 & 97,4 & 88,9 & 60,7 \\
\hline
\end{tabular}


MERCEDES VARONA ALABERN

PROPUESTAS PARA ELEVAR LOS DEFICIENTES NIVELES ACADÉMICOS MASCULINOS

\begin{tabular}{|l|c|c|c|c|c|c|c|c|c|c|}
\hline \multirow{2}{*}{ PAísES } & 2000 & 2005 & 2010 & 2014 & 2015 & \multicolumn{5}{|c|}{2016} \\
\cline { 2 - 12 } & $25-64$ & $25-64$ & $25-64$ & $25-64$ & $25-64$ & $25-64$ & $25-34$ & $35-44$ & $45-54$ & $55-64$ \\
\hline Latvia & 83,2 & 84,4 & 88,6 & 86,7 & 87,8 & 88,7 & 86,7 & 85,2 & 92,8 & 89,9 \\
\hline Luxembourg & 60,9 & 65,9 & 77,7 & 82 & 74,6 & 78,8 & 86,6 & 84,3 & 73,4 & 68,4 \\
\hline Mexico & 29,1 & 28,2 & 32,1 & 35,1 & 35,7 & 36,6 & 46,7 & 36 & 32,4 & 25,7 \\
\hline Netherlan. & 64,9 & 71,8 & 73 & 75,9 & 76,4 & 77,1 & 85,8 & 81,5 & 75,9 & 66 \\
\hline Norway & - & 77,2 & 80,6 & 81,9 & 82,4 & 82,2 & 81,2 & 85,6 & 82,2 & 79,7 \\
\hline Poland & 79,9 & 85,1 & 88,5 & 90,5 & 90,8 & 91,3 & 94,2 & 93,5 & 91,7 & 85,8 \\
\hline Portugal & 19,4 & 26,5 & 31,9 & 43,3 & 45,1 & 46,9 & 69,5 & 57,4 & 37,3 & 26 \\
\hline Slovak Rp. & 83,8 & 87,9 & 91 & 90,8 & 91,3 & 91,7 & 93,4 & 94,2 & 92,2 & 86,6 \\
\hline Slovenia & 74,8 & 80,3 & 83,3 & 85,7 & 86,8 & 87,3 & 94,1 & 92,6 & 84,6 & 78,6 \\
\hline Spain & 38,6 & 48,8 & 52,9 & 56,6 & 57,4 & 58,3 & 65,3 & 67 & 55,9 & 42,7 \\
\hline Sweden & 77,6 & 83,6 & 86,3 & 81,6 & 82 & 82,7 & 83,1 & 85,6 & 85,3 & 76 \\
\hline Switzerl. & 83,9 & 85,2 & 85 & 87,2 & 87,3 & 87,4 & 91,4 & 88,4 & 86,2 & 83,7 \\
\hline Turkey & 23,3 & 28,1 & 31,2 & 35,6 & 37 & 38,5 & 54,7 & 39 & 25,6 & 22,7 \\
\hline UK & 62,6 & 66,8 & 75,1 & 79,2 & 79,6 & 80,7 & 87,5 & 84,7 & 77,7 & 72,3 \\
\hline USA & 87,4 & 87,8 & 89 & 89,6 & 89,5 & 90,1 & 91,5 & 89,5 & 89,4 & 90,2 \\
\hline
\end{tabular}

Fuente: NATional Center Education Statistics (2017).

La repetición de curso se encuentra estrechamente ligada al fracaso escolar, siendo también superior entre los chicos que entre las chicas, dado que tan solo un $53,6 \%$ de los varones de cuarto de secundaria tiene la edad que le corresponde, frente al 64,2\% de las chicas (Choi y Calero, 2013: 567), contribuyendo a los bajos niveles académicos de la población española y en concreto, de los varones (Tabla 4), quienes han ido retrocediendo a lo largo del tiempo en relación a las féminas. La Tabla 3 refleja muy claramente la precaria situación académica de la población española en relación al resto de países de la OCDE, ya que solo el 58,3\% posee titulación de bachillerato o formación profesional equivalente. La economía del siglo XXI requiere de una población altamente formada y flexible para irse adaptando a los cambios constantes. Esta imprescindible formación es inaccesible en la actualidad en España para demasiados y pone en riesgo el futuro democrático de nuestro país, ya que la equidad, la estabilidad y el desarrollo dependen del nivel formativo de la población y de su capacidad para crear riqueza.

La superioridad de las féminas también está muy presente al otro lado del charco. Al ocaso del siglo XIX, las mujeres norteamericanas ya superaban a los varones en la obtención del diploma de high school (titulación equivalente a bachillerato o formación profesional) debido en parte a que ambos sexos compartían aula regentada por profesoras (Goldin, 1998). Esta supremacía continúa hasta nuestros 
días, dado que el $85,2 \%$ de las chicas se graduaron de high school, frente al 78,8\% de los chicos en 2013 (National Center Educational Statistics, 2016). En educación superior (Figura 1) el retroceso de los varones es más espectacular (Goldin, Katz y Kuziemko, 2006).

\section{TABLA 4}

Nivel de formación de la población adulta (25-64 años) en porcentajes. 2016

\begin{tabular}{|c|c|c|c|c|c|c|c|c|c|c|}
\hline $\begin{array}{c}\text { NIVELES DE } \\
\text { ESTUDIOS }\end{array}$ & \multicolumn{5}{|c|}{ MUJERES } & \multicolumn{5}{c|}{ HOMBRES } \\
\hline EDAD & $25-64$ & $25-34$ & $35-44$ & $45-54$ & $55-64$ & $25-64$ & $25-34$ & $35-44$ & $45-54$ & $55-64$ \\
\hline ESO & $39.1 \%$ & 28.9 & 28,4 & 42.3 & 59 & 44.3 & 40.6 & 37.4 & 45 & 55.6 \\
\hline High Schl. & 60.9 & 71.2 & 71.7 & 57.6 & 41 & 55.7 & 59.4 & 62.6 & 54.11 & 44.4 \\
\hline Superior & 38.4 & 47 & 49.5 & 33.6 & 21.7 & 33 & 35 & 38.8 & 31.5 & 24.8 \\
\hline
\end{tabular}

FuENTE: INSTITUTO NACIONAL DE ESTADística (2017)

Además de las grandes desventajas académicas de los varones, estos tienen hasta ocho veces más probabilidades de ser diagnosticados con hiperactividad; están sobrerrepresentados en la educación especial, y padecen mayores depresiones, ansiedad y suicidios. Del mismo modo, existe mayor número de varones con problemas de aprendizaje y disciplinarios (Kleinfeld, 2009).

A esto le tenemos que añadir que si en Occidente la esperanza de vida no ha dejado de crecer, en EE. uU. dicho índice se ha deteriorado desde 1999 para los varones de etnia blanca con edades comprendidas entre los 45 y los 54 años y con bajos niveles académicos (sin estudios superiores) como consecuencia de las drogas, el alcohol y el suicidio, descendiendo medio punto cada año, infiriendo que los problemas del sistema educativo norteamericano se ensañan especialmente con los varones (Case y Deaton, 2017). 
FIGURA 1

Evolución de la educación superior entre la población norteamericana por sexo. 1966-2019

\section{Percent of All College Degrees* Female vs. Male, 1966-2019}

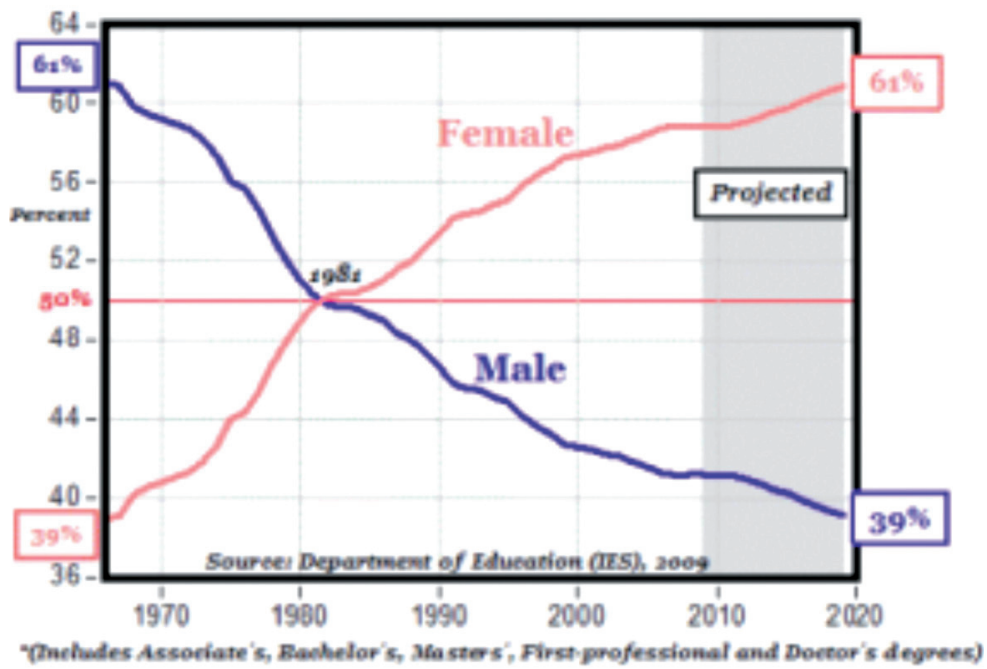

Nota: Female $=$ Mujeres; Male $=$ Hombres

FuENTE: Proposal for the White House Council on boys and men.

\section{CAUSAS DE LAS DEFICIENCIAS ACADÉMICAS DE LOS VARONES}

Las principales causas de los bajos rendimientos académicos masculinos se podrían resumir en:

- Diferencias evolutivas del ser humano.

- Diferencias en los procesos de aprendizaje de las niñas y de los niños.

- La ausencia del padre en el desarrollo de sus hijos.

- Desequilibrio en la composición del profesorado y parcialidad en su percepción.

\subsection{Diferencias evolutivas del ser humano}

Todas las células humanas tienen 23 pares de cromosomas, excepto los gametos sexuales que tienen la mitad, siendo uno de los pares el determinante de la identidad sexual femenina, XX, o masculina, XY. 
En la Figura 2 se observa la prominente diferencia de tamaño entre el cromosoma $\mathrm{x}$ y el $\mathrm{Y}$, disparidad debida a que el primero alberga alrededor de 1.100 genes (Ross, Grafham, Coffey, Scherer, Mclay, Muzny, et al., 2005), aproximadamente diez veces más que el cromosoma «Y» (National Human Genome Research Institute, 2015), y este patrón se reproduce en todas y cada una de las células del varón, confiriéndole, conjuntamente con la carencia de las características propias del par de cromosomas femeninos xx, su identidad sexual masculina.

En el reino animal, las hembras de los mamíferos cuentan con 2 cromosomas $\mathrm{X}$ para la reposición de genes y para equilibrar su expresión, manteniendo uno de ellos inactivo para evitar duplicidades. Sin embargo, estudios recientes (Carrel y Willard, 2005) han descubierto que un 15\% del cromosoma femenino humano $\mathrm{X}$ etiquetado como inactivo escapa a esta inactividad, contribuyendo a las diferencias biológicas entre mujeres y hombres, mientras que otro $10 \%$ se manifiesta de forma dispar entre las mujeres, confiriéndoles características distintas y distanciando a las mujeres de la mayor homogeneidad entre los varones.

FIGURA 2

Par de cromosoma masculino XY

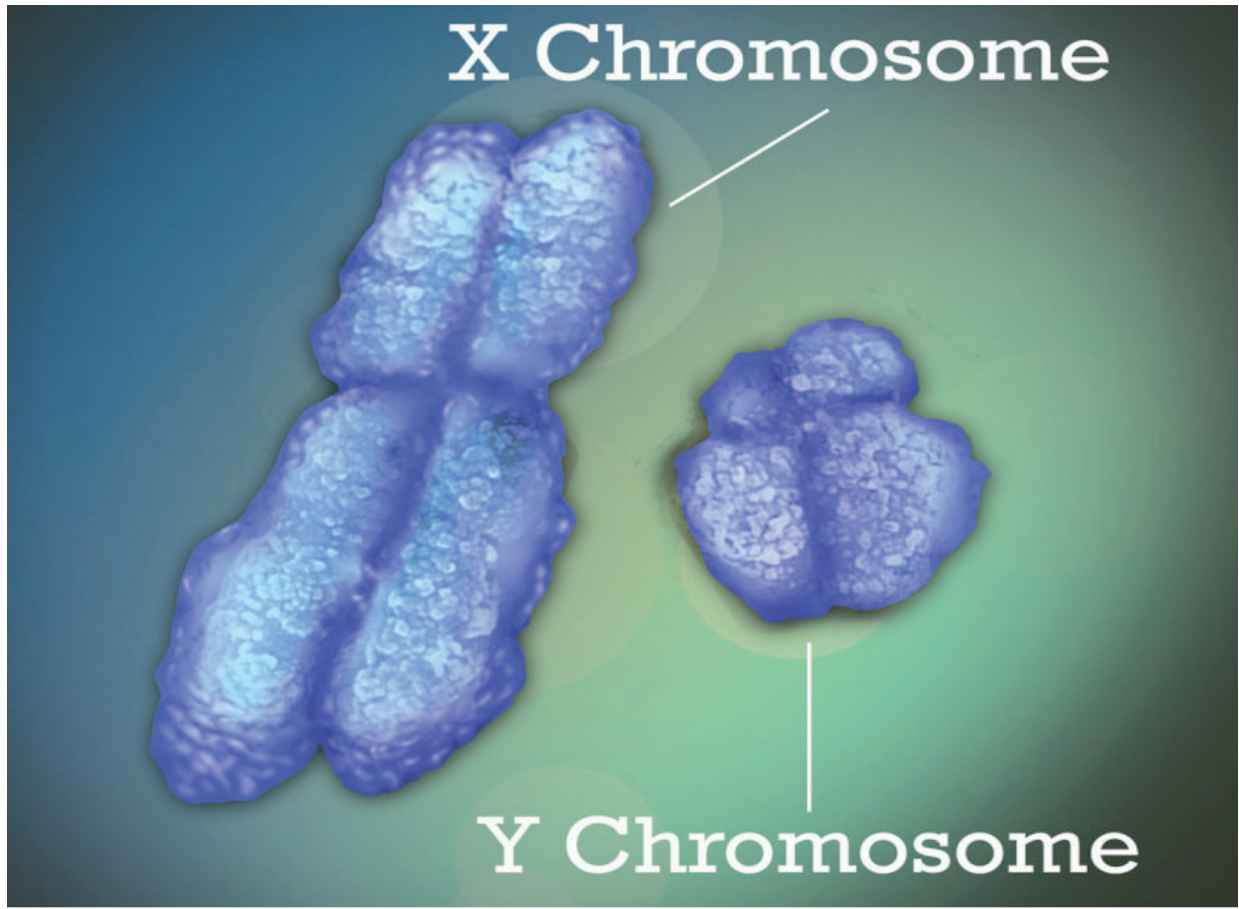


Estas notables diferencias entre ambos sexos quedan reflejadas en que las disimilitudes entre mujeres y hombres representan el 1,5\% de su genoma, coincidiendo en el 98,5\% restante (Page, 2013), frente al 99,8\%, entre el mismo sexo (Sabatini, 2016). A primera vista un $1,5 \%$ podría parecer una diferencia despreciable, no obstante, esta toma gran relevancia cuando se tienen en cuenta las elevadas confluencias entre el genoma humano y el de los animales: con el gato, por ejemplo, es del 90\% (Pontius, Mulikin y Smith, 2007), y con el chimpancé, es del 96\% (Varki y Altheide, 2015). Dichas similitudes son debidas a que todos los seres vivos e inertes tienen un origen común, por este motivo, están todos conectados (Martínez y Arsuaga, 2002). A estas diferencias fundamentales se les debe añadir que los genes se expresan de forma dispar en ambos sexos para determinar su diferente identidad sexual (Gershoni y Pietrokovski, 2017; Rosenblatt, 2015) y que, de los 20.000 genes humanos aproximados (National Human Genome Research Institute, 2007), 6.500 son exclusivamente femeninos o masculinos, es decir, una disparidad nada despreciable de más del 30\% (Sánchez-Serrano, 2017).

Para concluir este apartado señalar que la mayoría de especies del reino animal están divididas entre hembras y machos por la especialización dirigida a la optimización de los recursos con la finalidad de perpetuar la especie y de lograr una mayor sofisticación o complejidad en su desarrollo. Si los dos sexos estuvieran diseñados para realizar exactamente las mismas funciones, la naturaleza hubiera eliminado a uno de ellos, ya que los recursos son limitados y no hay lugar para la duplicidad (Martínez y Arsuaga, 2002).

\subsection{Diferencias en los procesos de aprendizaje de las niñas y de los niños}

La optimización de los recursos ha derivado en la diferenciación de los cerebros femeninos y masculinos que se complementan perfectamente, como se desprende de la Figura 3, y estas diferencias biológicas influyen directamente en el comportamiento dispar de ambos sexos y en su proceso de aprendizaje (Tunç, Solmaz, Parker, Satterthwaite, Elliott, Calkins et al., 2016).

La materia gris, formada principalmente por los cuerpos celulares, es más abundante en las mujeres, especialmente en las regiones relacionadas con el lenguaje (área de Broca y Wernicke) (Schlaepfer, Harris, Tien, Peng, Lee y Pearlson, 1995), mientras que la materia blanca, formada por los axones (las conexiones neuronales) es superior en los varones. De la Figura 3 también se desprende que existe mayor conectividad dentro de cada hemisferio en los varones para facilitar una respuesta más rápida, pero al mismo tiempo poseen menos recursos para redirigir un plan fallido. Las conexiones entre hemisferios son superiores en la mujer, lo que facilita la comunicación entre los procesos analíticos e intuitivos y la realización de "multitareas» (cambiar de tarea de forma rápida). Asimismo, se ha observado que el hipocampo (mediador entre la memoria y las emociones) y el cuerpo calloso (facilitador de la comunicación entre los 2 hemisferios) son de mayor tamaño en las mujeres, mientras que las áreas neurológicas del lenguaje están más desarrolladas 
en las niñas que en los niños. Asimismo, ambos sexos utilizan distintas partes del cerebro para realizar dichas tareas (Burman, Bitan y Booth, 2008). Sin dejar a las mujeres, las mayores cantidades de la hormona oxitocina que segregan refuerzan los vínculos afectivos y las sensibilizan hacia las necesidades de los demás, facilitando la maternidad y la empatía (Marlin, Mitre, D'Amour, Chao y Froemke, 2015; Gurian, 2011: 23, 27, 29). Paralelamente, las mujeres suelen controlar mejor sus impulsos y se arriesgan menos, gracias a las mayores cantidades de serotonina y las menores cantidades de testosterona, sintiéndose además menos cómodas en ambientes competitivos (Niederle y Vesterlund, 2010).

FIGURA 3

Diferencias entre el cerebro masculino y el femenino
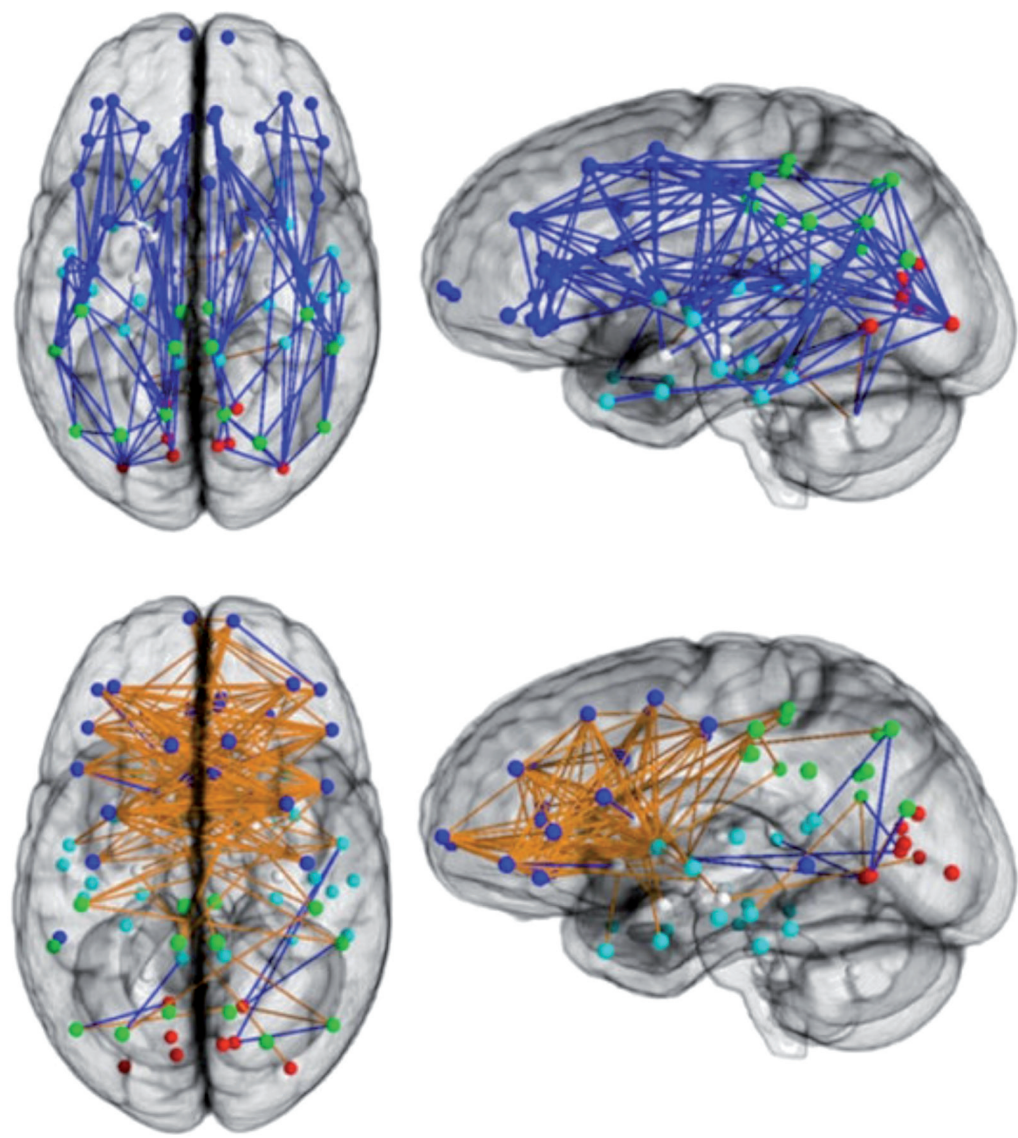

Nota. El primero es el cerebro masculino y el segundo el femenino.

Fuente: Gentileza de Ingalhalikar, Smith, Parker, Satterthwaite, Elliott, Ruparel, et al., 2014. 
Los cerebros femeninos y masculinos no solo son distintos (Ruigrok, Salimi-Khorshidi, Lai, Baron-Cohen, Lombardo, Tait et al., 2014) y funcionan de forma dispar, sino que se desarrollan a diferentes ritmos. El cerebro femenino alcanza su volumen máximo a los 10,5 años, frente a los 14,5 años del masculino. En la densidad de la materia gris la diferencia entre ambos sexos es de 1 año (Lenroot, Gogtay, Greenstein, Molloy, Wallace, Clasen et al., 2007), reflejándose en la disparidad académica y en el proceso de maduración de cada sexo. El cerebro de una niña de 2 años en el área del lenguaje equivale al de un niño de 5 años, porque mientras que en la primera esta zona cerebral se desarrolla pronto, al igual que la motricidad fina y la velocidad perceptiva, en los niños el desarrollo temprano se centra en las tareas viso-espaciales y la motricidad gruesa (Gil-Verona, Macías, Pastor, De Paz, Barbosa, Maniega et al., 2003).

Un estudio realizado en 2010 por Özçalişkan y Goldin-Meadow mostraba cómo las niñas recién nacidas (de 1 a 3 días de vida) imitaban mejor el movimiento de los dedos que los niños y esta diferencia persistía hasta los 5-6 años. La gesticulación es la primicia para el aprendizaje del lenguaje. En esta misma línea se encuentra el estudio de Connellan, Baron-Cohen, Wheelwright, Batki y Ahluwalia (2000), quienes observaron la reacción de los bebés de 1 día de vida al ver rostros femeninos u objetos mecánicos con rasgos femeninos. Las niñas se interesaron por los rostros femeninos, mientras que los niños eligieron los juguetes. Este estudio fue replicado con niños de 1 año, esta vez mostrando a los bebés videos de vehículos o de caras en movimiento. Los resultados fueron idénticos (Lutchmaya y Baron-Cohen, 2002). Además se analizó el contacto visual a esas tempranas edades y de nuevo las niñas mantuvieron más contacto visual con sus padres que los niños (Lutchmaya, Baron-Cohen y Raggatt, 2002). Las investigaciones de Todd, Barry y Thommessen (2016) añadieron que los bebés de 9 meses de edad eligieron los juguetes deacuerdo con su sexo. Asimismo, Alexander (2014) llegó a resultados similares al estudiar bebés de entre 3 y 8 meses de edad, las niñas miraban más las muñecas y los niños los camiones. Sin dejar a los recién nacidos, se observó que cuando a los bebés prematuros se les tarareaban canciones, las niñas recibían el alta antes que los niños (Sax, 2005: 16), todo ello demostrando que los vínculos afectivos y sociales están más desarrollados en las niñas, lo que les facilita una mejor adaptación al entorno escolar. Dichas diferencias afectan no solo las actitudes y comportamientos de ambos sexos, sino a sus capacidades para adquirir nuevos conocimientos y experiencias e integrarlas en sus vidas.

Por su parte, los varones gozan de mejores habilidades espaciales y mecánicas, y mejor razonamiento abstracto, expresándose mejor en lenguaje no verbal, por este motivo, pueden responder más despacio. Asimismo, obtienen mayores rendimientos cuando están sometidos a moderados niveles de estrés, gracias a la producción de adrenalina, aunque sean las féminas las que toleran mejor la presión a largo plazo (Berenbaum, Bryk y Beltz, 2012; Ingalhalikar, Smith, Parker, Satterthwaite, Elliott, Ruparel et al., 2014). Poseen igualmente elevadas cantidades de testosterona, potenciador de la agresividad, y bajos niveles del neurotransmisor 
serotonina, inhibidor de la misma y del impulso, confiriéndoles una agresividad natural (Cahill, 2005). Por este motivo, existen más varones que mujeres que cometen crímenes violentos, sufren alcoholismo, se suicidan, terminan en prisión o padecen déficit de atención (Gurian, 2011: 23). Consecuentemente, los chicos necesitan realizar mayor ejercicio físico, participar en actividades competitivas, marcarse retos y recibir reconocimiento para aumentar la dopamina, neurotransmisor necesario para la motivación y para sentirse valorado y arraigado (Keller, 2013). El deporte es también imprescindible para que ellas puedan funcionar bien, aunque suela estar infravalorado.

Todas estas diferencias biológicas dirigidas a que las niñas aprendan a comunicarse verbalmente antes que los niños condicionan el aprendizaje de estos últimos en el sistema educativo actual, a lo que se le tiene que añadir que el mayor tamaño de la amígdala en los varones, conjuntamente con la elevada cantidad de testosterona, les dificultan prestar atención, permanecer largos periodos de tiempo sentados, escuchar e interesarse por conceptos, atrayéndoles en cambio aquellos objetos físicos o mecánicos en movimiento o que ellos puedan manipular o explorar. Les atraen más la ciencia ficción o los libros de acción que otro tipo de género literario, utilizan mayor agresividad en sus juegos y tienden a llamar más la atención (Cahill, 2005). Todas estas diferencias son receta para el fracaso masculino en el sistema educativo actual.

\subsection{La ausencia del padre en el desarrollo de sus hijos}

El estilo educativo del padre difiere del de la madre, yendo dirigido a animar a sus hijos a resolver problemas por su cuenta, a ser más independientes, lo que les da seguridad, madurez y habilidades para la vida adulta (Paquette, 2004). Por este motivo, la figura paterna tiene especial relevancia en el rendimiento académico de los hijos varones y en su desarrollo (Jeynes, 2015). Cuando el padre infunde confianza y autonomía a los hijos varones hacia los dos años y medio de edad, esta influencia favorece un mayor éxito académico durante los 3 primeros cursos de primaria. Mientras que el apoyo materno en la autonomía de los hijos varones refuerza la autosuficiencia a la edad de 6 años, el apoyo del padre tiene mayor trascendencia, extendiéndose hasta tercero de primaria (Belsky, Bradley, Brownell, Burchinal, Campbell, Booth-LaForce et al., 2008).

Por otro lado, la relación negativa entre familias desestructuradas regentadas por la madre con los problemas escolares y sociales de los varones está ampliamente documentada (Qureshi y Ahmad, 2014). Una investigación llevada a cabo durante 10 años en EE. UU. puso de manifiesto que los niños de etnia negra que viven solo con la madre tienden a obtener peores resultados académicos, a abandonar prematuramente los estudios, a sufrir fracaso escolar y a tener mayores problemas disciplinarios que sus hermanas (Autor, Figlio, Karbownik, Roth y Wasserman, 2016). Del mismo modo, el escaso interés del padre por los estudios de su 
hijo reduce la posibilidad de que este salga de la pobreza en un $25 \%$, siendo los datos para las chicas en relación a sus madres equivalentes (Blanden, 2006: 15).

Resultados similares destacan las consecuencias negativas de la ausencia de la figura paterna en las aptitudes no cognitivas de los niños, atenuadas, sin embargo, en sus hermanas, sufriendo los hijos varones de familias monoparentales el doble de probabilidades de ser expulsados de la escuela durante el primer ciclo de secundaria (Bertrand y Pan, 2011). Investigadores españoles alcanzaron conclusiones análogas al constatar que la privación de la figura paterna provoca problemas académicos y conductuales en los hijos varones (Cantón, Cortés y Justicia, 2002). Igualmente, la ausencia del padre está ligada a actitudes antisociales en sus vástagos varones (Mitchell, McLanahan, Hobcraft, Brooks-Gunn, Garfinkel y Notterman, 2015), así como a conductas agresivas tanto verbales, como físicas, al carecer de un modelo positivo a quien imitar soportando igualmente un mayor riesgo de sufrir abusos (Rodríguez, Del Barrio y Carrasco, 2013). Por consiguiente, el porcentaje de familias monoparentales que reside en un barrio se encuentra estrechamente ligado al grado de pobreza y de delincuencia del mismo (Smith y Jarjoura, 1988).

La irremplazable figura del padre en el desarrollo de sus hijos y en concreto de sus hijos varones se manifiesta a muy temprana edad. Se ha observado que cuanto mayor sea la interacción de los bebés con su padre y, en especial, los de etnia negra, mayores habilidades mentales y psicomotoras poseerá su hijo, incluso a edades tan tempranas como a los 6 meses, es decir, la ausencia del padre puede disminuir la inteligencia de su hijo varón, porque las habilidades psicomotoras a temprana edad están asociadas con aumentos de la inteligencia (Pedersen, Rubenstein y Yarrow, 1979; Yogman, Kindlon y Earls, 1995; Nettle, 2008). Los beneficios de la presencia del padre fueron también constatados analizando los efectos que estos tenían en la recuperación de recién nacidos prematuros hospitalizados. Las visitas del padre acortaban la estancia en el hospital de los bebés, estos aumentaban de peso y mejoraban sus funciones psico-sociales y adaptativas (Levy-Shiff, Hoffman, Mogilner, Levinger y Mogilner, 1990).

La estrecha relación entre el padre y sus hijos varones es tan fundamental que la ausencia del padre perjudica con especial virulencia el organismo de sus vástagos, reduciendo la longitud de los telómeros de los cromosomas, responsables de la protección de los mismos y cuya deficiencia está asociada con enfermedades como el cáncer. Los chicos sufren tal anomalía un 40\% más que las chicas (Mitchell, McLanahan, Schneper, Garfinkel, Brooks-Gunn y Notterman, 2017).

A la luz de todas estas investigaciones parecería lógico que la sociedad y las autoridades pertinentes aunaran esfuerzos para apoyar a los padres y promover su responsabilidad con sus hijos. No obstante, los datos oficiales son alarmantes y nos revelan que el 23\% de los niños menores de 18 años en EE. UU. vive solo con la madre, alcanzando a casi 17 millones de niños. Esta cifra se eleva hasta el 50\% para los menores de etnia negra y hasta el $26 \%$ para los hispanos. No en vano son los colectivos más castigados por la pobreza y la violencia. El número de familias monoparentales en EE.UU. ha aumentando drásticamente del 8\% en 1960, al 23\% en 
2016, mientras que el porcentaje de menores que convive con los dos progenitores ha disminuido del 88\% en 1960, al 69\% actual (U.S. Census Bureau, 2016).

En España 2 millones aproximadamente de menores de 25 años viven solo con la madre ${ }^{1}$, representando alrededor del 17,4\% del total (Instituto Nacional de Estadística, 2015 y OECD, 2016). Si estimamos que la mitad de ellos son varones, la cifra de niños y jóvenes en riesgo de exclusión es muy elevada.

\subsection{Desequilibrio en la composición del profesorado y parcialidad en su percepción}

El 81\% de los maestros españoles de primaria son mujeres (Instituto Nacional de Estadística, 2017), disminuyendo ligeramente hasta el $72 \%$ cuando se le añade la educación secundaria, y coincidiendo con los datos de la mayoría de países de nuestro entorno. En 2005 las féminas copaban la profesión de maestro de primaria en Occidente, oscilando entre el 89\% en EE. UU. y el 78\% en Suiza y en secundaria oscilaba entre el 63\% en EE. UU. y el 45\% en Holanda. Esta descompensación se mantiene invariable en el tiempo, representando las mujeres el $85 \%$ de los profesores de primaria en la Unión Europea, mientras que en secundaria este porcentaje supuso el 64\% en 2014 (Eurostat, 2016).

Los desequilibrios en la composición del profesorado feminizan la enseñanza (Dee, 2005), privando a los niños de modelos masculinos a seguir y alejándose de la premisa integradora en donde se sustenta la coeducación. Ahondando en este tema, Ouazad y Page (2013) cuestionaron la imparcialidad del profesorado con los alumnos del sexo contrario, demostrando que las profesoras puntúan más bajo a sus alumnos varones, coincidiendo con la percepción que estos tienen de sus maestras. Por el contrario, las alumnas consideraban recibir un trato favorable por parte de sus profesores, cuando en realidad los maestros varones tendían a beneficiar a los alumnos de su mismo sexo. Siguiendo en esta línea, los profesores propenden a presuponer que los chicos tienen mejor nivel en matemáticas que las chicas y estos prejuicios perjudican los resultados académicos de las alumnas, obteniendo entre un $40 \%-75 \%$ menos de puntuación que los chicos en EE. UU. (Robinson, Lubienski y Copur, 2011).

Por otro lado, Myhill y Jones (2006) destacan que los chicos suelen ser percibidos como un problema, mientras que las chicas son presentadas como estudiantes modelo. Por este motivo, los chicos reciben mayor atención, pero esta es negativa, mientras que ellas reciben más atención positiva y esta percepción aumenta con la edad, contribuyendo todo ello a los bajos rendimientos académicos de los chicos. Sin embargo, los profesores no son conscientes de las diferencias de aprendizaje existentes entre ambos sexos, ni de la parcialidad con que tratan a sus alumnos

1. En España 1.541.700 hogares eran regentados solo por la madre en 2015. El tamaño medio de hogar monoparental es 2,35. Por consiguiente, $1,541.700 \times 1,35$ (excluyendo la madre) $=2.081 .295$ niños viven sólo con la madre. 
(Cornwell, Mustard y Van Parys, 2011). Por otro lado, el sesgo también está muy presente en los alumnos de educación superior, dado que otorgaron mejor puntación a los profesores en la educación a distancia cuando creían que eran varones (MacNell, Driscoll y Hunt, 2015).

Otro de los problemas de la carencia de profesorado masculino en la coeducación es el círculo vicioso que esta genera. Los varones consideran la educación una profesión femenina, por estar el profesorado compuesto mayoritariamente por mujeres y por los mejores resultados académicos de las chicas, alejándoles de la docencia y de la excelencia académica. A esto hay que añadir que en la coeducación la composición de las aulas puede incluso discriminar en número a uno de los dos sexos. Paralelamente, las desigualdades entre niñas y niños no solo no disminuyen en la coeducación, sino que aumentan, al incrementarse las desigualdades académicas y comportamentales en el aula. Por este motivo, los alumnos varones no son los únicos perjudicados por el sistema educativo actual, sino que las féminas pueden ver truncadas sus aspiraciones a carreras técnicas, es decir, al intentar eliminar las diferencias y barreras de la educación diferenciada, se han creado inadvertidamente otras que pueden alejar a las alumnas de las matemáticas, de la física, etc., como ocurre en EE. UU., y a los alumnos, de profesiones tales como la docencia (Norfleet James y Richards, 2003). Por otro lado, las chicas también se benefician de tener profesoras al mejorar sus rendimientos académicos (Lim y Meer, 2015), rindiendo además mejor cuando trabajan con otras chicas, reduciéndose su rendimiento a la mitad cuando su compañero es un varón (Stetson University).

\section{Evolución de la educación. Problemas Generados}

La expansión de la educación mixta tiene su origen a principios del xx, cuando Europa ofrecía educación secundaria principalmente a las élites económicas e intelectuales, mientras que EE. UU. comenzó a formar a todos los jóvenes, confiriéndoles una ventaja económica, social y política sin precedentes, integrando asimismo a las chicas y a los chicos en la misma aula por ser este modelo más económico y más práctico (Goldin y Katz, 2008: 11-13). El éxito educativo norteamericano ayudó a consolidar la educación mixta, derivando en la «estandarización" de la educación del alumnado, los cuales continúan siendo formados «en serie», con miras puestas en la economía industrializada del siglo pasado, necesitada de trabajadores disciplinados y uniformes, desdeñando de este modo la singularidad de cada alumno y sus misiones particulares. Por este motivo, las razones para el auge y difusión de la coeducación no residieron tanto en minuciosos estudios científicos que justificaran tal apreciación, sino en decisiones regidas más por la conveniencia, la ideología o la economía que por la excelencia, y estas decisiones han perjudicado principalmente y de forma muy categórica a los colectivos masculinos más vulnerables. A esto hay que añadir que la coeducación norteamericana ofrecía dos líneas de aprendizaje, una para las chicas dirigida hacia el mercado laboral o el hogar y la otra para los chicos encaminándolos hacia la educación superior (Madigan, 2009), 
infiriéndose que compartir aula no garantiza el acceso a la misma formación. Es decir, la educación mixta no asegura la equidad, como ha quedado demostrado. Asimismo, el descenso de los niveles académicos masculinos coincidió en España con la implantación de la educación mixta, y en eE. UU. con la aprobación de la ley Title IX of the Education Amendments Act of 1972, que excluía la educación diferenciada de la recepción de fondos públicos y eliminaba las dos vías de enseñanza. En 2006, se modificó dicha Enmienda para permitir crear centros diferenciados con fondos públicos.

La coeducación facilita la convivencia entre ambos sexos preparando a los alumnos para la vida adulta, allanando su colaboración para complementarse para completarse. Asimismo, en el mundo globalizado la diversidad será la norma que rija las relaciones sociales, por tanto, parecería lógico posicionarse a favor de que la escuela reflejara esta realidad y preparara a sus alumnos para ella, es decir, que en vez de separar los sexos, los uniera. Estos postulados teóricos que tienen tanta resonancia en la sociedad actual han producido efectos secundarios no deseados suficientemente importantes para eclipsar los beneficios que aportan. La realidad se impone a cualquier opinión o deseo, y los sistemas educativos actuales expulsan a un número demasiado elevado de varones, al uniformar los sistemas educativos y desestimar las características propias de cada individuo, haciéndose de esta forma necesaria una revisión educativa en profundidad.

\section{DisCUSIONES Y SOLUCIONES PARA MEJORAR LOS BAJOS NIVELES ACADÉMICOS DE LOS VARONES}

La importante diferencia genética del 1,5\% entre el genoma humano femenino y el masculino se manifiesta contundentemente desde el primer día de vida, afectando directamente los procesos de aprendizaje y de socialización tanto de las niñas como de los niños, y precisando tenerlos en consideración al diseñar cualquier sistema educativo. Esta evidencia se omite en la didáctica actual.

Por otra parte, a diferencia de los animales que albergan en sus genes la mayoría de conocimientos que necesitan para sobrevivir, el ser humano debe aprender, por imitación o estudio, casi todo de las generaciones anteriores a través de los libros, de los maestros, de la familia, de las tradiciones, de las costumbres, de la religión, etc. (Martínez y Arsuaga, 2002). Por este motivo, la carencia de referentes masculinos para emular afecta muy negativamente el desarrollo físico, psíquico y académico de los alumnos varones. También se ha comprobado como los niños presentan aptitudes, comportamientos, preferencias, intereses, etc. masculinos para relacionarse con el mundo exterior, y de esta forma, aprender, compartiendo su peculiar manera de ser con sus iguales, secundados por el padre y por los maestros varones, ayudándoles de este modo a reforzar su seguridad en sí mismos, es decir, ayudándoles a crecer y madurar (Hällgren, Dunkels y Frånberg, 2015: 60-61), reduciendo al mismo tiempo la sobrerrepresentación de estos en la educación especial (Piechura-Couture, Heins, y Tichenor, 2013). 
Consecuentemente, parece lógico deducir que el modelo teórico de coeducación, presentado como integrador y socializador, tiene en la práctica unas consecuencias no deseadas durante las primeras etapas educativas contrarias a estos objetivos, planteándose la necesidad de revisar dicho modelo y considerar la necesidad de incorporar la educación diferenciada, con profesores del mismo sexo que los alumnos durante las etapas de primaria y primeros cursos de secundaria, hasta que estos adquieran las habilidades necesarias para integrarse con las féminas, beneficiando también a estas últimas. Sin embargo, se ha comprobado que la educación diferenciada selectiva, dentro de un entorno mixto, puede ayudar a las chicas, pero no así a los chicos (Jackson, 2002).

Por otro lado, los graves problemas derivados de la carencia de la figura paterna en la vida de los niños, una realidad que va en aumento, podrían mitigarse en la escuela a través no sólo de una educación diferenciada, sino asignando mentores masculinos que les apartaran de la pobreza, la violencia, las drogas, la influencia de los medios, las bandas juveniles o del mundo virtual que ellos controlan, a diferencia del entorno escolar, donde son las chicas las que destacan, y, de esta forma, ayudarles a madurar, crecer, adquirir seguridad, sentirse valorados y lograr las habilidades necesarias para salir adelante. Asimismo, los profesores varones tendrán un efecto positivo en los chicos más vulnerables siempre y cuando el entorno sea diferenciado, sensible hacia sus necesidades y compense la ausencia física, cognitiva, social y psicológica de la figura paterna.

La transición del sistema actual a otro más sensible a las necesidades escolares de los varones requerirá de la contratación de profesores varones, especialmente para la etapa educativa de primaria, y la formación de mentores, existiendo asimismo material didáctico para afrontar tal cometido (Stetson University).

\section{CONCLUSIONES}

Las diferencias biológicas, de desarrollo y de maduración entre ambos sexos perjudican principalmente a los varones en los sistemas académicos actuales. El modelo educativo vigente basado en la coeducación aporta beneficios reconocidos al normalizar las relaciones entre chicas y chicos, preparándoles para la vida adulta y potenciando a las primeras, pero paradójicamente, ha ocasionado una serie de problemas con graves consecuencias para el individuo y para la sociedad. Por este motivo, durante aquellas etapas de la infancia en que la coeducación resulta negativa, es decir, hasta que las diferencias académicas, de desarrollo y de maduración se vayan atenuando y no obstaculicen ni lastren la educación mixta, un modelo alternativo de educación ayudaría al alumnado masculino. De este modo, los estudiantes, bien entrada la adolescencia, ya han adquirido la suficiente seguridad en sí mismos para comenzar a salir de su mundo homogéneo e interesarse por el otro sexo. Uno de los problemas de la educación es la estandarización del aprendizaje, es decir, la imposición de currículos y sistemas sin tener en cuenta las necesidades y características propias de cada individuo. Del mismo modo, la 
tendencia innata a asociarse con iguales para adquirir seguridad y para aprender por imitación es vulnerada por una escuela uniformadora y teórica, alejada de la evidencia. La infancia y la juventud son etapas necesarias para la maduración del individuo y para ello necesitan un ambiente amoroso, estable, de apoyo, seguro y estimulante, el cual no se consigue enteramente en la coeducación. Los varones son los primeros en sufrir las consecuencias del sistema educativo actual, especialmente aquellos privados de la figura paterna durante su infancia, padeciendo niveles académicos deficitarios, conductas disruptivas y mayor probabilidad de caer en la pobreza, repercutiendo todo ello negativamente en la totalidad de la sociedad. Por consiguiente, una revisión del sistema educativo español para mejorar los bajos niveles académicos de la sociedad es necesaria, dado que la economía del siglo XXI requiere de una población altamente formada y flexible para irse adaptando a los cambios constantes. Esta imprescindible formación es inaccesible en la actualidad para demasiados y pone en riesgo el futuro democrático de nuestro país, ya que la equidad, la estabilidad y el desarrollo dependen del nivel formativo de la población y de su capacidad para crear riqueza.

\section{REFERENCIAS BIBLIOGRÁFICAS}

Alexander, G. M. (2014). Postnatal Testosterone Concentrations and Male Social Development. Front Endocrinol, 5, 15.

Autor, D.; Figlio, D.; Karbownik, K.; Roth, J. y Wasserman, M. (2016). Family Disadvantage and the Gender Gap in Behavioral and Educational Outcomes. NBER, 22267.

Belsky, J.; Booth-LaForce, C.; Bradley, R.; Brownell, C. A.; Burchinal, M. y Campbell, S. B. et al. (2008). Mothers' and fathers' support for child autonomy and early school achievement. Developmental Psychology, 44 (4), 895-907.

Berenbaum, S. A.; Bryk, K. L. y Beltz, A. M. (2012). Early androgen effects on spatial and mechanical abilities: evidence from congenital adrenal hiperplasia. Behavioral Neuroscience, 126 (1), 86-96.

Bertrand, M. y Pan, J. (2011). The Trouble with Boys: Social Influences and the Gender Gap in Disruptive Behavior. NBER, 17541.

Blanden, J. (2006). «Bucking the trend»: What enables those who are disadvantaged in childhood to succeed later in life? Department for Work and Pensions, Working Paper, 31. Descargado el 28 de enero de 2018. http://dera.ioe.ac.uk/7729/1/WP31.pdf.

Burman, D. D.; Bitan, T. y Booth, J. R. (2008). Differences in Neural Processing of Language Among Children. Neuropsychologia, 46 (5), 1349-1362.

Cahill, L. (2005). His Brain, her Brain. Scientific American, 292 (5), 40-47.

Cantón Duarte, J.; Cortés Arboleda, M. R. y Justicia Díaz, M. D. (2002). Las consecuencias del divorcio en los hijos. Psicopatología Clínica, Legal y Forense, 2 (3), 47-66.

Carrel, L. y Willard, H. F. (2005). X-inactivation profile reveals extensive variability in $\mathrm{X}$-linked gene expression in females. Nature, 434, 400-404.

Case, A. y Deaton, A. (2017). Mortality and morbidity in the 21st century. Brooking Papers on Economic Activity.

Casquero Tomás, A. y Navarro Gómez, M. L. (2010). Determinantes del abandono escolar temprano en España: un análisis. Revista de Educación, número extraordinario 
2010, 191-223. Descargado el 13 de febrero de 2018. http://www.ugr.es/ fjjrios/pce/ media/4e-Re2010_08Genero.pdf.

Connellan, J.; Baron-Cohen, S.; Wheelwright, S.; Batki, A. y Ahluwalia, J. (2000). Sex differences in human neonatal social perception. Infant Behavior \& Development, 23 (1), 113-118.

Cornwell, C. M.; Mustard, D. B. y Van Parys, J. (2011). Non-Cognitive Skills and the Gender Disparities in Test Scores and Teacher Assesments: Evidence from Primary School. IZA, 5973. Descargado el 26 de enero de 2018. https://d-nb.info/1015948006/34.

Choi de Mendizábal, A. y Calero Martínez, J. (2013). Determinantes del riesgo de fracaso escolar en España en PISA-2009 y propuestas de reforma. Revista de Educación, 362, $562-593$.

Dee, T. (2005). Teachers and the Gender Gaps in Student Achievement. NBER, 11660.

Eliot, L. (2013). Single-sex education and the brain. Sex Roles, 69 (7-8).

Eurostat (2016). Women teachers largely over-represented in primary education in the EU. No. 189/2016. Descargado el 19 de enero de 2018. http://ec.europa.eu/eurostat/ documents/2995521/7672738/3-04102016-BP-EN.pdf/9f0d2d04-211a-487d-87c3-0a5f$7 \mathrm{~d} 6 \mathrm{~b} 22 \mathrm{c}$.

Gershoni, M. y Pietrokovski, S. (2017). The landscape of sex-differential transcriptome and its consequent selection in human adults. BMC Biology, 15.

Gil-Verona, J. A.; Macías, J. A.; Pastor, J. F.; De Paz F.; Barbosa, M.; y Maniega, M. A. et al. (2003). Diferencias sexuales en el sistema nervioso humano. Una revisión desde el punto de vista psiconeurobiológico. International Journal of Clinical and Health Psychology, 3 (2), 351-361.

Goldin, C. (1998). America's Graduation from High School: The Evolution and Spread of secondary schooling in the twentieth century. Journal of Economic History, 58 (2), 345-374.

Goldin, C. y Katz, L. F. (2008). The Race between Education and Technology. Cambridge: Harvard University Press.

Goldin, C.; Katz, L. F. y Kuziemko, I. (2006). The homecomming of American College Woman: the Reversal of the college gap. Journal of economic perspectives, 20 (4), 133156.

Gurian, M. (2011). Boys and girls learn differently 2011. San Francisco: Jossey-Bass.

Hällgren, C.; Dunkels, E. y Frånberg, G. M. (2015). Invisible Boys. Exploring the making of boys. Umeå: Print \& Media, Umeå University.

Halpern, D. F.; Benbow, C. P.; Geary, D. C.; Gur, R. C.; Hyde, J. S. y Gernsbacher, M. A. (2007). The Science of Sex Differences in Science and Mathematics. Psychological Science in the Public Interest, 8 (1), 1-51.

Ingalhalikar, M.; Smith, A.; Parker, D.; Satterthwaite, T. D.; Elliott, M. A.; y Ruparel, K. et al. (2014). Sex differences in the structural connectome of the human brain. PNAS, 111 (2), 823-828.

Instituto Nacional de Estadística (2015). Encuesta Continua de Hogares. Descargado el 11 de enero de 2018. http://www.ine.es/prensa/np965.pdf.

Instituto Nacional de Estadística (2017). Educación. 3.10. Mujeres en el profesorado por enseñanza que imparten. Descargado el 23 de enero de 2018. 3_10_Mujeres_en_el_p. pdf.

Jackson, C. (2002). Can Single-sex Classes in Co-educational Schools Enhance the Learning Experiences of Girls and/or Boys? An Exploration of Pupils' Perceptions. British Educational Research Journal, 28 (1), 37-48. 
Jeynes, W. H. (2015). A Meta-Analysis: The Relationship Between Father Involvement and Student Academic. Urban Education, 50 (4), 387-423.

Jordan-Young, R. y Rumiati, R. I. (2011). Hardwired for Sexism? Approaches to Sex/Gender in Neuroscience. Neuroethics, 5 (3).

Keller, G. (2013). Pink, Brain, Blue Brain. Descargado el 25 de enero de 2018. http://eduexcel.co.za/wp-content/uploads/2013/03/PINK-BRAIN-BLUE-BRAIN- 11- page-summary. pdf.

Kleinfeld, J. (2009). The State of American Boyhood. Gender Issues, 26, 113-129.

Lenroot, R. K.; Gogtay, N.; Greenstein, D. K.; Molloy Wells, E.; Wallace, G. L.; y Clasen, L. S. et al. (2007). Sexual dimorphism of brain developmental trajectories during childhood and adolescence. Neuroimage, 36 (4), 1065-1073.

Levy-Shiff, R.; Hoffman, M. A.; Mogilner, S.; Levinger, S. y Mogilner, M. B. (1990). Fathers' hospital visits to their preterm infants as a predictor of father-infant relationship and infant development. Pediatrics, 86 (2), 289-293.

Lim, J. y Meer, J. (2015). The Impact of Teacher-Student gender matches: Random Assignment Evidence from South Korea. NBER, 21407. Descargado el 9 de enero de 2018. http://www.nber.org/papers/w21407.pdf.

Lutchmaya, S. y Baron-Cohen, S. (2002). Human sex differences in social and non- social looking preferences, at 12 months of age. Infant Behavior and Development, 25 (3), 319-325.

Lutchmaya, S.; Baron-Cohen, S. y Raggatt, P. (2002). Foetal testosterone and eye contact in 12- month-old human infants. Infant Behavior \& Development, 25 (3), 327-335.

MacNell, L.; Driscoll, A. y Hunt, A. N. (2015). What's in a Name: Exposing Gender Bias in Student Ratings of Teaching. Innovative Higher Education, 40 (4), 291-303.

Madigan, J. C. (2009). The education of women and girls in the United States: A historical perspective. Advances in Gender and Education, 11-13.

Marlin, B. J.; Mitre, M.; D'Amour, J. A.; Chao M. V.; y Froemke, R. (2015). Oxytocin enables maternal behaviour by balancing cortical inhibition. Nature, 520, 499-504.

Martínes, I. y Arsuaga, J. L. (2002). Amalur: Del átomo a la mente. Madrid: Ediciones Temas de Hoy.

Mitchell, C.; McLanahan, S.; Hobcraft, J.; Brooks-Gunn, J.; Garfinkel, I. y Notterman, D. (2015). Family Structure Instability, Genetic Sensitivity and Child Wellbeing. American Journal of Sociology, 120 (4), 1195-1225.

Mitchell, C.; McLanahan, S.; Schneper, L.; Brooks-Gunn, J.; Garfinkel, I. y Notterman, D. (2017). Father Loss and Child Telomere Length. Pediatrics, 140 (2).

Ministerio de Educación, Cultura y Deporte (2017). Sistema estatal de indicadores de la educacion 2017. Descargado el 17 de enero de 2018. https://www.mecd.gob.es/ $\mathrm{dms} / \mathrm{mecd} /$ servicios-al-ciudadano-mecd/estadisticas/educacion/indicadores-publicaciones-sintesis/sistema-estatal- indicadores/SEIE_2017.pdf.

Ministerio de Educación y Ciencia. CIDE (1992). Las Desigualdades en la Educación en España. Descargado el 25 de enero de 2018. https://www.uam.es/personal_pdi/stmaria/jmurillo/documentos/Desigualdades.pdf.

Myhill y Jones (2006). She doesn't shout at no girls': pupils' perceptions of gender equity in the classroom. Cambridge Journal of Education, 36 (1), 99-113.

National Center for Education Statistics NCES. (2016). Digest of education 2016. Tabla. 219.40. Descargado el 11 de enero de 2018. https://nces.ed.gov/programs/digest/d16/ tables/dt16_219.40.asp. 
National Human Genome Research Institute (2015). Facts about the X Chromosome that all mothers - all females-carry! Descargado el 18 de enero de 2018. https://www.genome. gov/27561568/facts-about-the-x-chromosome/.

National Human Genome Research Institute (2007). A Guide to your Genome. Descargado el 17 de enero de 2018. https://www.genome.gov/pages/education/allaboutthehumangenomeproject/guideto yourgen ome07_vs2.pdf.

Nettle, D. (2008). Why do some dads get more involved than others? Evidence from a large British cohort. Evolution and Human Behavior, 29 (6), 416-423.

Niederle, M. y Vesterlund, L. (2010). Explaining the Gender Gap in Math Test Scores: The Role of Competition. Journal of Economic Perspectives, 24 (2), 129-144.

Norfleet James, A. (2015). Teaching the male brain. Thousand Oaks, CA: Corwin.

Norfleet, J. A. y Richards, H. C. (2003). Escaping stereotypes: Educational attitudes of male alumni of single-sex and coed schools. Psychology of Men E Masculinity, 4 (2), 136-148.

OECD Family Database (2016). SF1.1: Family size and household composition. Descargado el 23 de enero de 2018. https://www.oecd.org/ELS/FAMILY/SF_1_1_FAMILY_SIZE_AND_ COMPOSITION.PDF.

Ouazad, A. y Page, L. (2013) Students' Perceptions of Teacher Biases: Experimental economic in school. Journal of Public Economics, 105, 116-130.

Özçalişkan, S. y Goldin-Meadow, S. (2010). Sex differences in language first appear in Gesture. Developmental science, 13 (5).

Page, D. C. (2013). Why sex really matters. TEDxBecaonStreet. Consultado el 27 de febrero de 2018. https://www.youtube.com/watch?v=nQcgD5DpVlQ.

Paquette, D. (2004). Theorizing the Father-Child Relationship: Mechanisms and Developmental Outcomes. Human Development, 47, 193-219.

Pedersen, F. A.; Rubenstein, J. L. y Yarrow, L. J. (1979) Infant Development in Father-Absent Families. Journal of Genetic Psychology, 135, 51-61.

Piechura-Couture, K.; Heins, E. y Tichenor, M. (2013). The Boy Factor: Can Single- Gender Classes Reduce the Over-Representation of Boys in Special Education? College Student Journal, 47 (2), 235-243.

Pontius, J. U.; Mulikin, J. C. y Smith, D. R. (2007). Initial sequence and comparative analysis of the cat genome. Genome Research, 17 (11), 1675-1689.

Porposal for a White House Council on boys and men (2014). Descargado el 21 de febrero de 2018. http://whitehouseboysmen.org/the-proposal/the-education-of-our-sons.

Qureshi, M. S. y Ahmad, A. (2014). Effects of Father Absence on Children's Academic Performance. Journal of Educational, Health and Community Psychology, 3 (1), 1-6.

Robinson, J. P.; Lubienski, S. T. y Copur, Y. (2011). The Effects of Teachers' Gender- Stereotypical Expectations on the Development of the Math Gender Gap. Society for Research on Educational Effectiveness. Fall.

Ross, M. T.; Grafham, D. V.; Coffey, A. J.; Scherer, S.; Mclay, K.; y Muzny, D. et al. (2005). The DNA sequence of the human $\mathrm{x}$ chromosome. Nature, 434, 325-337.

Rodríguez, M. A.; Del Barrio, M. V. y Carrasco, M. A. (2013). Agresión física y verbal en hijos de familias monoparentales divorciadas y biparentales: El efecto moderador del sexo de los hijos. Revista de Psicopatología y Psicología Clínica, 18 (2), 119-127.

Ruigrok, A. N. V.; Salimi-Khorshidi, G.; Lai, M. C.; Baron-Cohen, S.; Lombardo, M. V. y Tait et al. (2014). A meta-analysis of sex differences in human brain structure. Neuroscience E Biobehavioral Reviews, 39, 34-50. 
Sabatini, R. (2016). How to read the genome and build a human being. TED2016. Descargado el 7 de febrero de 2018. https://www.ted.com/talks/riccardo_sabatini_how_to_read_ the_genome_and_build_a_h uman_being\#t-225365.

Sax, L. (2005). Why gender matters: What parents and teachers need to know about the emerging science of sex differences. New York: Doubleday.

Sánchez-Serrano, I. (2017). Gender-Specific Medicine's Imminent Coming of Age. Gender and the Genome, 1 (4).

Schlaepfer, T. E.; Harris, G. J.; Tien, A. Y.; Peng, L.; Lee, S. y Pearlson, G. D. (1995). Structural differences in the cerebral cortex of healthy female and male subjects: a magnetic resonance imaging study. Phychiatric research, 61 (3), 129-135.

Smith, D. A. y Jarjoura, G. R. (1988). Social Structure and Criminal Victimization. Journal of Research in Crime and Delinquency, 25 (1), 27-52.

Stetson University. Single-Gender Education: A Handbook for Teachers and Principals. Descargado el 20 de febrero de 2018. http://www.stetson.edu/artsci/education/hollis-institute/media/GenderManualFInaL[1].pdf.

Todd, B. K.; Barry, J. A. y Thommessen, S. A. O. (2016). Preferences for 'Gender-typed' Toys in Boys and Girls Aged 9 to 32 Months. Infant and Child Development, 26 (3).

Tunç, B.; Solmaz, B.; Parker, D.; Satterthwaite, T. D.; Elliott, M. A.; y Calkins, M. E. et al. (2016). Establishing a link between sex-related differences in the structural connectome and behaviour. Philosophical Transactions of the Royal Society B: Biological Sciences, 371 (1688).

U. S. Census Bureau Reports (2016). The Majority of Children Live With Two Parents. CB16192. Descargado el 5 de febrero de 2018. https://www.census.gov/newsroom/pressreleases/2016/cb16-192.html.

Varki, A. y Altheide, T. K. (2015). Comparing the human and chimpanzee genomes: Searching for needles in a haystack. Genome Research, 15 (2), 1746-1758.

Yogman, M. W.; Kindlon, D. y Earls, F. (1995). Father involvement and cognitive/behavioral outcomes of preterm infants. Journal of the American Academy of Child and Adolescent Psychiatry, 34 (1), 58-66. 